Final Abstract on CRADA \# BNL-C-93-04 between BNL and eV Products II-IV, Inc.

The objective of the project was to pursue research and development of CdTe and $\mathrm{CdZnTe}$ materials for room temperature radiation detectors. The goal was to significantly improve the existing material synthesis, growth and processing techniques based on the better understanding of the formation of crystal defects during crystal growth and processing as well as the influence of the defects and impurities on the electrical characteristics of the detector materials.

The work resulted in a better insulating coating for enveloping the detector crystal and reducing the leakage current through the crystal surface, an improved etching technique to get better and reliable crystal surface finish before the deposition of the metal electrical contacts, an improved deposition technique for the metal contacts on the crystal surface. A better understanding of the origins of polarization phenomena, i.e. detector performance deterioration below room temperatures was also achieved. 
Final Report on CRADA \# BNL-C-93-04 between BNL and eV Products II-IV, Inc.

Title of Project:

Principal Investigator:

Industrial Partner:
$\mathrm{CdTe} / \mathrm{CdZnTe}$ Characterization and Detector Development

Kelvin G.Lynn

Low Energy Particle-Solid Interaction (LEPIS) Group

Tel: (516) 2823710

Elgin E. Eissler and Carl J. Johnson

eV Products Division of II-VI Inc.

375 Saxonburg Boulevard, Saxonburg, PA 16056

Tel: (412) 3525288

Fax: (412) 3524980

\section{Description of Research:}

The objective of the project was to pursue research and development of CdTe and $\mathrm{CdZnTe}$ materials for room temperature radiation detectors. These materials exhibit high sensitivity for 10 to $500 \mathrm{keV}$ photons and the detectors can be operated at low bias voltages of 15 to 300 volts. The High Pressure Bridgman growth technique employed by $\mathrm{eV}$ Products/II-VI allows the production of high quality crystals of large size. To fully exploit the advances of this growth technique and enhance the market potential of the material improvement in the yield and resolution must be realized.

Unknown defect properties both at the surface and in the bulk of these crystals are the most important hindrance for the full-scale commercialization of $\mathrm{CdTe} / \mathrm{CdZnTe}$ detectors. The goal is to significantly improve the existing material synthesis, growth and processing techniques based on the better understanding of the formation of crystal defects during crystal growth and processing as well as the influence of the defects and impurities on the electrical characteristics of the detector materials.

Two major goals has been set for this project:

1) Provide quantitative information about defect properties in the CdTe and $\mathrm{CdZnTe}$ materials and relate their effects to carrier transport properties and on detector performances; 
2) Develop reliable and high-quality metal contacts for $\mathrm{CdTe} / \mathrm{CdZnTe}$ detectors. Examine electrochemical, evaporation and sputtering contact deposition for various surface treatments of the crystals.

$\mathrm{eV}$ Products/II-IV provided BNL with series of CdTe and CdZnTe crystals grown from various starting material and under various conditions. The crystals were underwent a series of etching and contact deposition procedures and their performance was characterized by one or more of the following characterization techniques: transient charge technique, current-voltage, temperature dependent pulse height measurements. Some samples were also characterized by the thermoelectric effect/thermally stimulated current spectrometer currently being developed at Brookhaven. Data on the detector performance, bulk and metal-semiconductor interface carrier traps, trapping/detrapping of electrons and holes, and carrier transport has been accumulated and related to the material growth parameters and crystal processing. A study to reduce the surface leakage current has been also performed and an insulating coating technique has been developed.

\section{Significant Accomplishments:}

Significant accomplishments include:

1) a better insulating coating for enveloping the detector crystal and reducing the leakage current through the crystal surface,

2) an improved etching technique to get better and reliable crystal surface finish before the deposition of the metal electrical contacts,

3) an improved deposition technique for the metal contacts on the crystal surface,

4) a better understanding of the origins of polarization phenomena, i.e. detector performance deterioration at temperatures below room temperatures, and the growth parameters influencing it.

\section{Significant Problems: None}

\section{Industry Benefits Realized:}

The improved crystal etching, contact deposition and crystal packing improved the reliability of the radiation detectors manufactured from these crystals. The chemical etching, sputter deposition and surface coating technique of the crystals have been adopted and are now used in production by $\mathrm{eV}$ Products. The better control of the crystal processing steps reduced the trial-and-error in the detector fabrication by reducing the manufacturing time and costs and resulting more reliable detector performance. The 
improved reliability and longer lifetime of the detectors, on the long run, lead to consumer satisfaction and enhanced sales.

\section{Laboratory Benefits Realized:}

Significant amount of knowledge and experience has been accumulated on the surface conditions and states of CdTe and CdZnTe crystals af ter etching, on the Schottky barrier formation upon metal contact deposition both by chemical and physical techniques. Some insight has been gained on the role of surface and interface defects on the charge collection process. The collective experience gained have a beneficial positive feedback to the semiconductor defect studies of the positron group. The defect characterization techniques under development, in particular the thermoelectric effect/ thermally stimulated current spectroscopies are planned to be used in a broad range of other semiconducting/insulating materials.

\section{Recommended Follow-on Work:}

One important disadvantageous property of the CdTe and CdZnTe based materials is the so-called polarization phenomenon. This is essentially a deterioration of the detector performance if the crystal is cooled below room temperature. According to the existing interpretations of the polarization phenomenon it is related to charge trapping close to the metal contacts at the crystal surface. We have performed systematic studies of the temperature dependence of the detector pulse height spectra in order to determine the voltage, temperature and time dependence of the polarization of crystals grown under various conditions. Further studies are highly recommended to understand the physical mechanism of polarization and to develop a crystal processing and contact deposition technique which significantly suppress the polarization phenomenon. A preliminary study on ZnSe crystals, also produced by II-VI Inc., as possible high temperature $\left(100^{\circ} \mathrm{C}\right)$ radiation detector has shown promising results. Further characterization studies are highly desirable to evaluate such a new application of this material.

\section{Potential Benefits from Pursuing Follow-on Work:}

A significant advantage of this follow-on work would be the development of detectors with a much wider operation temperature range, and reduced operation voltage requirements which would greatly expand the range of applicability of CdTe and particularly CdZnTe materials as radiation detectors. The application of $\mathrm{ZnSe}$ crystals would provide unique detectors which would tolerate much higher ambient temperatures than any radiation detector available today. 